\title{
SPLITTING CRITERIA AND EXTENSION TYPES ${ }^{1}$
}

\section{REINHOLD BAER AND CHRISTINE WILLIAMS}

Introduction. Following the lead given by Galois theory it appears natural to term extensions $G$ and $H$ of the group $N$ equivalent extensions, if there exists an isomorphism of $G$ upon $H$ which leaves invariant every element in $N$. But if $G$ is an extension of $N$, and if $S$ is some group, then it will, in general, be impossible to assert the equivalence of the extensions $G$ and $G \oplus S$ of $N$ in spite of the fact that these two extensions of $N$ do not differ in any interesting feature. Thus one may feel that the concept of equivalence provides too narrow a principle of classification of extensions. With this in mind we say that the extension type of the extension $G$ of $N$ is contained in the extension type of the extension $H$ of $N$, if there exists a homomorphism of $G$ into $H$ which leaves invariant every element in $N$ and which maps normal subgroups of $G$ upon normal subgroups of $H$; and we say that the extensions $G$ and $H$ of $N$ belong to the same extension type if the type of $G$ is contained in the type of $H$ and the type of $H$ is contained in the type of $G$. In this way we obtain a partially ordered set of extension types of $N(\S 3)$.

It is clear that the extensions $G$ and $G \oplus S$ of $N$, mentioned before, belong to the same extension type of $N$; and more generally $G \oplus S$ and $G \oplus T$ belong to the same extension type of $N$, if $G$ is an extension of $N$. In order to obtain criteria for the validity of the converse we need splitting criteria asserting the existence of complements which are normal subgroups. Since hardly any such criteria are available in the literature (see Baer $[2]^{2}$ for references) we devote the first section to the derivation of such criteria which may be of interest independent of the applications in \$3. In these applications there exists always a normal subgroup whose elements are left invariant by the endomorphisms under consideration. This makes it possible to weaken the hypotheses usually needed; and a systematic account of these phenomena is given in $\$ 2$. In an appendix we show that finitely generated groups are not isomorphic to proper quotient groups, if their normality preserving endomorphisms split.

1. Normality and uniformity of splitting. We consider $M$-groups $G$ where the composition of elements in $G$ is addition $x+y$ and where

Received by the editors May 15, 1948.

1 This work has been done partially under a contract with the Office of Naval Research; however, ONR assumes no responsibility for the material in this paper.

${ }^{2}$ Numbers in brackets refer to the bibliography at the end of the paper. 
the operators in $M$ are left-multipliers so that the effect of applying $m$ in $M$ on $g$ in $G$ is indicated by $m g$. Addition and multiplication meet the customary requirements. We note that no composition of the elements in $M$ is defined.

Terms like $M$-subgroup and $M$-endomorphism and so on are used in the customary way. In particular we note that we have $m(g \eta)$ $=(m g) \eta=m g \eta$ for $m$ in $M, g$ in $G$ and $\eta$ an $M$-endomorphism. The kernel of $\eta$ will be denoted by $K(\eta)$ and the join of the ascending chain of kernels $K\left(\eta^{i}\right)$ is the radical $R(\eta)$. Kernel and radical are $M$-admissible normal subgroups of $G$.

An $M$-admissible subgroup $C$ of $G$ is called a complement of the endomorphism $\eta$ of $G$, if $C=C \eta$ and if every coset of $G$ modulo $R(\eta)$ contains one and only one element in $C$. Then $\eta$ induces essentially the same automorphism in $G / R(\eta)$ and in the complement $C$. If it so happens that $\eta$ possesses one and only one complement, then we designate the uniquely determined complement of $\eta$ by $C(\eta)$.

We shall be interested in finding complements that are normal subgroups too, since then the group $G$ is represented as the direct sum $R \oplus C$ of the radical and complement of $\eta$. That this is by no means a rare occurrence may be seen from the following criterion.

If the $M$-endomorphism $\eta$ of $G$ possesses one and only one complement $C(\eta)$, and if $\eta$ commutes with every inner automorphism of $G$, then $C(\eta)$ is a normal subgroup of $G$.

Proof. If $g$ is an element in $G$, then $-g+R(\eta)+g=R(\eta)$, since $R(\eta)$ is a normal subgroup of $G$. Hence every coset of $G$ modulo $R(\eta)$ contains one and only one element in $-g+C(\eta)+g$. But $\eta$ commutes with inner automorphisms. Hence

$$
[-g+C(\eta)+g]_{\eta}=-g+C(\eta) \eta+g=-g+C(\eta)+g .
$$

Thus $-g+C(\eta)+g$ is a complement of $\eta$. But $\eta$ possesses only one complement. Hence $C(\eta)=-g+C(\eta)+g$. This proves the normality of $C(\eta)$.

In our applications in $\S 3$ we shall not be able to make use of this criterion, since then we shall not be assured of the hypothesis that $\eta$ commutes with the inner automorphisms, as the endomorphisms considered there are products of homomorphisms. Thus we turn now to the derivation of another type of criterion which assures at the same time the existence of a complement.

A subgroup $S$ of $G$ is $(M, \eta)$-admissible, if $M S \leqq S$ and $S \eta \leqq S$. The subgroups $L_{i}$ form an ascending Loewy chain for $\eta$, if

(a) $0=L_{0} \leqq L_{1} \leqq \cdots \leqq L_{i} \leqq L_{i+1} \leqq \cdots$;

(b) every $L_{i}$ is a normal $(M, \eta)$-admissible subgroup $L$ of $G$; 
(c) every $L_{i+1} / L_{i}$ is the sum of (a finite or infinite number of) minimal normal $(M, \eta)$-admissible subgroups of $G / L_{i}$.

Here we note that $J / L_{i}$ is a minimal normal $(M, \eta)$-admissible subgroup of $G / L_{i}$ if, and only if, $J$ is a normal $(M, \eta)$-admissible subgroup of $G$ such that $L_{i}<J$ and such that $L_{i}<S \leqq J$ together with normality and $(M, \eta)$-admissibility of the subgroup $S$ imply $S=J$.

At present it does not matter whether such a chain terminates after a finite number of steps or not. Since equality of successive terms is permitted, we may always assume these chains to be formally infinite.

Lemma 1. Let $\eta$ be an $M$-endomorphism of the $M$-group $G$. If $J$ is a minimal normal $(M, \eta)$-subgroup of $G$, then either

(a) $J \eta=0$ or

(b) $\eta$ maps $J$ isomorphically upon $J \eta$.

Proof. Since $K(\eta)$ is an $(M, \eta)$-subgroup which is normal in $G$ and $K(\eta) \cap J \leqq J$, either

(a) $K(\eta) \cap J=J$ so that $\eta$ maps every element of $J$ into zero or

(b) $K(\eta) \cap J=0$ so that $\eta$ induces an isomorphism of $J$ onto $J \eta$.

A particular type of $M$-endomorphism will be of importance in our considerations. We shall say that the $M$-endomorphism $\eta$ is a normality preserving $M$-endomorphism of $G$ if $\eta$ maps normal $M$-subgroups of $G$ upon normal $M$-subgroups of $G$.

LEMмa 2. If $S$ is the sum of minimal normal $(M, \eta)$-subgroups of $G, S \cap R(\eta)=S \cap K(\eta)$; there exists one and only one normal $(M, \eta)$ subgroup $C$ such that $S=C \oplus[S \cap R(\eta)]$. Furthermore, if $\eta$ is a normality preserving $M$-endomorphism of $G, C=C \eta=S \eta$.

Proof. Define $C$ to be the sum of all minimal normal $(M, \eta)$-subgroups $J$ of $G$ contained in $S$ with $J \eta \neq 0$. Then since $S$ is the sum of the minimal normal $(M, \eta)$-subgroups contained in it, $S=C$ $+[S \cap K(\eta)]$.

If $x$ is an element of $R(\eta) \cap C, x$ is in some direct sum $J_{1} \oplus \cdots \oplus J_{n}$, where the $J_{i}$ are minimal normal $(M, \eta)$-subgroups with $J_{i} \eta \neq 0$; hence $x=x_{1}+\cdots+x_{n}$ with $x_{i}$ in $J_{i}$. Since $x$ belongs to $R(\eta)$, there is a positive integer $m$ such that $0=x \eta^{m}=x_{1} \eta^{m}+\cdots+x_{n} \eta^{m}$; but $x_{i} \eta^{m}$ is in $J_{i}$ and the sum of the $J_{i}$ is a direct sum so that $0=x_{1} \eta^{m}$ $=\cdots=x_{n} \eta^{m}$. Since it follows from Lemma 1 that $\eta$ (and hence $\eta^{m}$ ) induces an isomorphic mapping of $J_{i}$, this implies that $x_{1}=\ldots$ $=x_{n}=0$ and therefore, $x=0$. Hence $C \cap R(\eta)=0$. Since $S \cap K(\eta)$ $\leqq S \cap R(\eta)$, we deduce from the Dedekind law that $S \cap R(\eta)$ 
$=[S \cap R(\eta)] \cap[C+(S \cap K(\eta))]=[S \cap R(\eta) \cap C]+[S \cap K(\eta)]=S$ $\cap K(\eta)$. Thus $S=C \oplus[S \cap R(\eta)]$.

That $C$ is the only normal $(M, \eta)$-admissible subgroup such that $S=C \oplus[S \cap R(\eta)]$ is a consequence of the fact that $C$ contains every minimal normal $(M, \eta)$-admissible subgroup which is not part of $S \cap R(\eta)$.

If $\eta$ is normality preserving, $J \eta=J$ for a minimal normal $(M, \eta)$ subgroup $J$ contained in $C$. Hence if $J \leqq C, J=J \eta \leqq C \eta$ so that $C \leqq C \eta$ and $C=C \eta$.

An example shows that for the last result, that is, that $S=S \eta$ $\oplus[S \cap R(\eta)]$, it is necessary to assume that $\eta$ is a normality preserving $M$-endomorphism of $G$.

EXAMPLE 1. Let $S$ be a simple group which possesses an isomorphic proper subgroup $R$ (for instance, $S$ can be taken as the group of all finite even permutations of the positive integers, and $R$ as the subgroup which consists of all permutations of $S$ which leave 1 fixed; see Schreier-Ulam [1]). Let $M$ be void, and let $\eta$ be the endomorphism which maps $S$ isomorphically onto $R$.

Then $S$ is a minimal normal $(M, \eta)$-subgroup of itself and $K(\eta)=0$ but $S \not \neq S \eta=R$.

Proposition 1. If the $L_{i}$ form an ascending Loewy chain of $(M, \eta)$ admissible subgroups of $G$, then $L_{i} \cap R(\eta)=L_{i} \cap K\left(\eta^{i}\right)$ and $\eta$ induces an isomorphism of $L_{i} \eta^{i}$ upon $L_{i} \eta^{i+1}$. If $\eta$ is normality preserving, $L_{i}=L_{i} \eta^{i} \oplus\left[L_{i} \cap R(\eta)\right]$ and $L_{i} \eta^{i}$ is the one and only complement of the endomorphism which is induced in $L_{i}$ by $\eta$.

Proof. We show first that $L_{i} \cap R(\eta)=L_{i} \cap K\left(\eta^{i}\right)$. By Lemma 2, $L_{1} \cap R(\eta)=L_{1} \cap K(\eta)$; assume that $L_{i-1} \cap R(\eta)=L_{i-1} \cap K\left(\eta^{i-1}\right)$. Since $L_{i} / L_{i-1}$ is the sum of minimal normal $(M, \eta)$-subgroups of $G / L_{i-1}$, by Lemma $2 L_{i} / L_{i-1}=C_{i} / L_{i-1} \oplus R_{i} / L_{i-1}$, where $R_{i}$ consists of all $x$ in $L_{i}$ such that $x \eta$ is in $L_{i-1}$; and $R_{i}$ contains every $x$ in $L_{i}$ such that $x \eta^{j}$ is in $L_{i-1}$ for some $j$. Suppose that $x$ is in $L_{i} \cap R(\eta)$; then $x \eta^{m}=0$ for some $m$ and hence $x$ is in $R_{i}$. But this implies that $x \eta$ is in $L_{i-1}$ $\cap R(\eta) \leqq K\left(\eta^{i-1}\right)$. Thus $x \eta^{i}=(x \eta) \eta^{i-1}=0$ and $x$ is in $K\left(\eta^{i}\right)$. Therefore, $L_{i} \cap R(\eta)=L_{i} \cap K\left(\eta^{i}\right)$.

It is clear that $\eta$ maps $L_{i} \eta^{i}$ homomorphically upon $L_{i} \eta^{i+1}$. Suppose that $x_{i} \eta^{i}$ is in $L_{i} \eta^{i}$ with $x_{i}$ in $L_{i}$ and that $\left(x_{i} \eta^{i}\right) \eta=0$; then $x_{i}$ is an element of $L_{i} \cap R(\eta)=L_{i} \cap K\left(\eta^{i}\right)$ so that $x_{i} \eta^{i}=0$. Hence $\eta$ maps $L_{i} \eta^{i}$ isomorphically upon $L_{i} \eta^{i+1}$.

For the remainder of the proof we assume that $\eta$ is a normality preserving $M$-endomorphism of $G$. By Lemma $2, L_{1} \eta^{2}=L_{1} \eta$; we use induction to show that $L_{i} \eta^{i+1}=L_{i} \eta^{i}$ for $i=1,2,3, \cdots$. 
Assume that $L_{i-1} \eta^{i}=L_{i-1} \eta^{i-1}$. Then $L_{i} / L_{i-1}=C_{i} / L_{i-1} \oplus R_{i} / L_{i-1}$, where $R_{i}$ is the subgroup of all elements $x$ in $L_{i}$ such that $x \eta$ belongs to $L_{i-1}$ and where $\left[L_{i} / L_{i-1}\right] \eta=\left[C_{i} / L_{i-1}\right] \eta=C_{i} / L_{i-1}$ by Lemma 2. Hence $C_{i}=L_{i} \eta+L_{i-1}=C_{i} \eta+L_{i-1}, \quad C_{i} \eta^{i-1}=L_{i} \eta^{i}+L_{i-1} \eta^{i-1}=L_{i} \eta^{i}$ $+L_{i-1} \eta^{i}=L_{i} \eta^{i}$; on the other hand, $C_{i} \eta^{i-1}=C_{i} \eta^{i}+L_{i-1} \eta^{i-1}=C_{i} \eta^{i}$ $+L_{i-1} \eta^{i}=C_{i} \eta^{i}$. Hence $L_{i} \eta^{i}=C_{i} \eta^{i-1}=C_{i} \eta^{i}=L_{i} \eta^{i+1}$. Thus we have shown that $\eta$ (and its powers) induce automorphisms in every $L_{i} \eta^{i}$.

We prove next that $L_{i}=L_{i} \eta^{i} \oplus\left[R(\eta) \cap L_{i}\right] . L_{i} \eta^{i}, R(\eta) \cap L_{i}$ are normal subgroups and $L_{i} \eta^{i} \cap\left[R(\eta) \cap L_{i}\right]=L_{i} \eta^{i} \cap K\left(\eta^{i}\right)=0$, since $\eta^{i}$ induces an automorphism of $L_{i} \eta^{i}$. Hence it suffices to show that $L_{i}=L_{i} \eta^{i}+\left[R(\eta) \cap L_{i}\right]$.

Let $x_{i}$ be in $L_{i}$; since $L_{i} \eta^{i}=L_{i} \eta^{2 i}$, there exists a $y_{i}$ in $L_{i}$ such that $x_{i} \eta^{i}=\left[y_{i} \eta^{i}\right] \eta^{i}$ and $x_{i}=y_{i} \eta^{i}+\left(-y \eta^{i}+x_{i}\right)$. Since $\left(-y \eta^{i}+x_{i}\right) \eta^{i}=-x_{i} \eta^{i}$ $+x \eta^{i}=0,-y \eta^{i}+x_{i}$ is in $R(\eta) \cap L_{i}$ and $L_{i}=L_{i} \eta^{i} \oplus\left[R(\eta) \cap L_{i}\right]$.

Finally we show that $L_{i} \eta^{i}$ is the one and only complement of $\eta$ in $L_{i}$. We have already shown that $L_{i} \eta^{i}$ is a complement of $\eta$ in $L_{i}$. Suppose $C^{\prime}$ is any complement of $\eta$ in $L_{i}$. If $x^{\prime}$ is in $C^{\prime}$ then $x^{\prime}=x_{i} \eta^{i}+z$, with $x_{i}$ in $L_{i}$, and $z$ in $R(\eta) \cap L_{i}=K\left(\eta^{i}\right) \cap L_{i}$; hence $x^{\prime} \eta^{i}=x_{i} \eta^{2 i}$ so that $C^{\prime}=C^{\prime} \eta^{i} \leqq L_{i} \eta^{i}$; but since $C^{\prime}$ has one element in each coset of $L_{i}$, modulo $L_{i} \cap R(\eta), C^{\prime}=L_{i} \eta^{i}$.

THEOREM 1. If $\eta$ is a normality preserving $M$-endomorphism of the $M$-group $G$, and if there exists an ascending Loewy chain of normal $(M, \eta)$-subgroups such that $G$ is their join, then $G=C(\eta) \oplus R(\eta)$, where $C(\eta)$ is the unique normal complement of $\eta$. Furthermore, if $N$ is a normal $(M, \eta)$-subgroup of $G, N=[N \cap C(\eta)] \oplus[N \cap R(\eta)]$.

Proof. Let $0=S_{0} \leqq S_{1} \leqq \cdots \leqq S_{i} \leqq \cdots$ be an ascending Loewy chain of normal $(M, \eta)$-subgroups of $G$ and let $N=\sum_{i=1}^{\infty} S_{i}$. By Proposition $1, S_{i}=S_{i} \eta^{i} \oplus\left[S_{i} \cap R(\eta)\right]$. If $x$ is an element of $N \cap R(\eta)$, $x$ is some $S_{i} \cap R(\eta)$ so that $N \cap R(\eta)=\sum_{i=1}^{\infty}\left[S_{i} \cap R(\eta)\right]$. Let $Q=\sum_{i=1}^{\infty} S_{i} \eta^{i}$; since $S_{i} \eta^{i} \cap R(\eta)=0$ for each $i, Q \cap R(\eta)=0$. Furthermore, $S_{i} \leqq S_{i} \eta^{i}+[N \cap R(\eta)] \leqq Q+[N \cap R(\eta)] \leqq N$ for each $i$; therefore, $N=Q \oplus[N \cap R(\eta)]$. Also $S_{i} \eta^{i}=S_{i} \eta^{i+1} \leqq Q \eta$ for each $i$ so that $Q \eta=Q$.

Now let $0=L_{0} \leqq L_{1} \leqq \cdots \leqq L_{i} \leqq \cdots$ be an ascending Loewy chain of normal $(M, \eta)$-subgroups of $G$ such that $G=\sum_{i=1}^{\infty} L_{i}$. Then if $C(\eta)=\sum_{i=1}^{\infty} L_{i} \eta^{i}, G=C(\eta) \oplus R(\eta)$ and $C(\eta)$ is a complement of $\eta$. Let $N$ be a normal $(M, \eta)$-subgroup of $G$; then $0=N \cap L_{0}=L_{0}^{\prime}$ $\leqq N \cap L_{1}=L_{1}^{\prime} \leqq \cdots \leqq N \cap L_{i}=L_{i}^{\prime} \leqq \cdots$ is an ascending chain of normal $(M, \eta)$-subgroups of $G$ with $N=\sum_{i=1}^{\infty} L_{i}^{\prime}$. Also $L_{i+1}^{\prime} / L_{i}^{\prime}$ $=N \cap L_{i+1} /\left[N \cap L_{i+1}\right] \cap L_{i} \cong\left(L_{i}+\left[N \cap L_{i+1}\right]\right) / L_{i} \leqq L_{i+1} / L_{i}$; this isomorphy between $L_{i+1}^{\prime} / L_{i}^{\prime}$ and the normal $(M, \eta)$-subgroup 
$L_{i}+\left[L_{i+1} \cap N\right] / L_{i}$ of $G / L_{i}$ maps normal subgroups of $G / L_{i}^{\prime}$ upon normal subgroups of $G / L_{i}$. Hence $L_{i+1}^{\prime} / L_{i}^{\prime}$ is the sum of minimal normal $(M, \eta)$-subgroups of $G / L_{i}^{\prime}$ and the $L_{i}^{\prime}$ form an ascending Loewy chain. Therefore, as we showed above, $N=\sum_{i=1}^{\infty} L_{i}^{\prime} \eta^{i}$ $\oplus[N \cap R(\eta)]$. But $\sum_{i=1}^{\infty} L_{i}^{\prime} \eta^{i}=\sum_{i=1}^{\infty}\left[N \cap L_{i}\right] \eta^{i} \leqq N \cap \sum_{i=1}^{\infty} L_{i} \eta^{i}$ $=N \cap C(\eta)$ and we deduce from the Dedekind law that

$$
\begin{aligned}
N \cap C(\eta) & =\left[\sum_{i=1}^{\infty} L_{i}^{\prime} \eta^{i}+[N \cap R(\eta)]\right] \cap[N \cap C(\eta)] \\
& =\sum_{i=1}^{\infty} L_{i}^{\prime} \eta^{i}+[[N \cap R(\eta)] \cap[N \cap C(\eta)]] \\
& =\sum_{i=1}^{\infty} L_{i}^{\prime} \eta^{i} .
\end{aligned}
$$

Hence $N=[N \cap C(\eta)] \oplus[N \cap R(\eta)]$.

Assume that $C^{\prime}$ is a normal complement of $G$. Then $C^{\prime}=C^{\prime} \cap C(\eta)$, since $C^{\prime} \cap R(\eta)=0$; but this implies that $C^{\prime} \leqq C(\eta)$, which shows that $C^{\prime}=C(\eta)$. Thus $C(\eta)$ is the unique normal complement of $\eta$.

If the $M$-endomorphism $\eta$ of the group $G$ possesses a complement, then $\eta$ is said to split $G$. If $S$ is an $M$-subgroup of $G$, and if $S \eta \leqq S$, then $\eta$ induces an $M$-endomorphism in $S$. If this induced endomorphism splits $S$, for every $(M, \eta)$-admissible subgroup $S$ of $G$, then we say that $\eta$ is a uniformly splitting endomorphism of $G$. Elsewhere a characterization of uniformly splitting endomorphisms has been given (Baer [2, Theorem A]); and it has been shown there that uniformly splitting endomorphisms possess one and only one complement (Baer [2, Corollary to Theorem A]).

The criterion for splitting given in Theorem 1 assures splitting of normal subgroups only so that this result cannot be used; and we can prove only the uniqueness of normal complements.

REMARK 1. Elsewhere (Baer [2, p. 515]) one may find an example of an endomorphism of an abelian operator group which does not split in spite of the fact that this group is the $(\omega+1)$ th term of its ascending Loewy chain. Thus our hypothesis on the length of the Loewy chain cannot be weakened in general.

We offer one application of the preceding results. If $G$ is an $M$ group, then we define inductively the normal $M$-subgroups $L_{i}(G)$ of $G$ by the rules:

$L_{0}(G)=0, L_{i+1}(G) / L_{i}(G)$ is the sum of all the minimal normal $M$-subgroups of $G / L_{i}(G)$.

It is easily seen that the $L_{i}(G)$ form a well defined ascending chain of characteristic and normal $M$-subgroups of $G$ and that every normality preserving $M$-endomorphism of $G$ maps every $L_{i}$ into itself.

Theorem 2. Suppose that the $M$-group $G$ meets the following re- 
quirements:

(a) Every element in $G$ belongs to at least one $L_{i}(G)$.

(b) $L_{i+1}(G) / L_{i}(G)$ is the sum of a finite number of minimal normal $M$-subgroups of $G / L_{i}(G)$.

Then normality preserving $M$-endomorphisms of $G$ possess unique normal complements.

Proof. The normal $M$-subgroups of $G$ situated between $L_{i}(G)$ and $L_{i+1}(G)$ satisfy the double chain condition, by (b). If $\eta$ is a normality preserving $M$-endomorphism of $G$, then $L_{i}(G) \eta \leqq L_{i}(G)$. Consequently we may construct, by intercalation of terms between $L_{i}(G)$ and $L_{i+1}(G)$, an ascending chain of $(M, \eta)$-admissible normal subgroups $S_{i}$ of $G$ with the following properties: $S_{i+1} / S_{i}$ is either 0 or a minimal normal $(M, \eta)$-subgroup of $G / S_{i}$; every element in $G$ belongs to at least one $S_{i}$. Then the $S_{i}$ form an ascending Loewy chain of normal $(M, \eta)$-subgroups of $G$ with join $G$; and now it is clear how to deduce Theorem 2 from the preceding results.

REMARK 2. It is not difficult to verify that Theorem C of Baer [2], if applied only on operator groups whose admissible subgroups are normal, will be a special case of Theorem 2 .

2. Almost periodicity on a subgroup. The $M$-endomorphism $\eta$ of the $M$-group $G$ is said to be almost periodic on the $M$-subgroup $S$ of $G$, if

(a) $S \eta \leqq S$ and

(b) there exists to every element $s$ in $S$ a positive integer $n=n(s)$ such that $s=s \eta^{n}$.

If $\eta$ is almost periodic on the normal $M$-subgroup $N$ of $G$, then $\eta$ induces an $M$-endomorphism in $G / N$. It is our object in the present section to relate the splitting properties of this induced endomorphism with those of $\eta$ itself.

LEMMA 1. If the $M$-endomorphism $\eta$ of the $M$-group $G$ is almost periodic on the normal $M$-subgroup $N$ of $G$, then

$$
N \cap R(\eta)=0
$$

and $[N \oplus R(\eta)] / N$ is the radical of the endomorphism induced by $\eta$ in $G / N$.

Proof. If $x$ belongs to $N \cap R(\eta)$, then there exist positive integers $i$ and $n$ such that $x \eta^{i}=0$ and $x \eta^{n}=x$. Then $x=x \eta^{i n}=0$. Thus $N \cap R(\eta)$ $=0$.

It is clear that $[N+R(\eta)] / N$ is part of the radical $R / N$ of the endomorphism which $\eta$ induces in $G / N$. If furthermore $x$ belongs to 
$R$, then there exists a positive integer $i$ such that $(N+x) \eta^{i}=0$; and this is equivalent to saying that $x \eta^{i}$ belongs to $N$. Consequently there exists a positive integer $n=n\left(x \eta^{i}\right)$ such that $x \eta^{i}=x \eta^{i+n}$. This implies $x \eta^{i}=x \eta^{i+i n}$. Let $y=x-x \eta^{i n}$. Then $y \eta^{i}=0$ so that $y$ belongs to $R(\eta)$. Furthermore $x \eta^{i n}=x\left(\eta^{i}\right)^{n}$ belongs to $N$, since $x \eta^{i}$ belongs to $N$, and since $N$ admits $\eta$. Hence $x=y+x \eta^{i n}$ belongs to $R(\eta)+N$. This shows that $R=R(\eta)+N$; and this completes the proof.

Proposition 1. Suppose that the $M$-endomorphism $\eta$ of the $M$-group $G$ is almost periodic on the normal $M$-subgroup $N$ of $G$. Then $C$ is a complement of $\eta$ if, and only if, $N \leqq C$ and $C / N$ is a complement of the endomorphism induced by $\eta$ in $G / N$.

Proof. Assume first that $C$ is a complement of $\eta$. If $x$ is an element in $N$, then there exists one and only one element $c$ in $C$ such that $x \equiv c$ modulo $R(\eta)$. Consequently there exists a positive integer $i$ such that $x \eta^{i}=c \eta^{i}$. Since $x$ is in $N$, there exists a positive integer $n$ such that $x=x \eta^{n}$. Hence $x=x \eta^{n i}=c \eta^{i n}$ belongs to $C$, since $C$ admits $\eta$. Thus we have shown that $N \leqq C$.

From $N \leqq C$ we infer by Dedekind's law that

$$
C \cap[N+R(\eta)]=N+[C \cap R(\eta)]=N,
$$

since the complement $C$ meets $R(\eta)$ in 0 . It follows now from Lemma 1 that 0 is the cross cut of $C / N$ and the radical of the endomorphism which $\eta$ induces in $G / N$.

From $C+N+R(\eta)=C+R(\eta)=G$ we infer that $G / N$ is the sum of $C / N$ and of the radical of the endomorphism induced in $G / N$ by $\eta$; and now it is easily verified that $C / N$ is a complement of the endomorphism induced by $\eta$ in $G / N$.

Assume now conversely that $N \leqq C$ and that $C / N$ is a complement of the endomorphism which is induced by $\eta$ in $G / N$. Since complement and radical have only 0 in common, it follows from Lemma 1 that

$$
N=C \cap[N+R(\eta)]=N+[C \cap R(\eta)]
$$

by Dedekind's law. Hence $C \cap R(\eta) \leqq N$ and it follows from Lemma 1 that

$$
C \cap R(\eta)=C \cap R(\eta) \cap N=0 .
$$

Since the sum of radical and complement is the full group, it follows from Lemma 1 that

$$
G=C+[N+R(\eta)]=C+R(\eta),
$$

since $N \leqq C$. Thus we have shown that every coset of $G$ modulo 
$R(\eta)$ contains one and only one element in $C$. Since the endomorphism induces an automorphism in the complement, it follows that

$$
C \eta+N=C \text {. }
$$

Thus there exist to every element $x$ in $C$ elements $y$ and $z$ in $C$ and $N$ respectively such that $x=y \eta+z$. Since $z$ is in $N$, there exists a positive integer $n$ such that $z=z \eta^{n}$. Since $N$ admits $\eta$, and since $z$ is in $N, z \eta^{n-1}$ belongs to $N$ too. Since $N \leqq C$, this implies that $y+z \eta^{n-1}=c$ is an element in $C$. Hence

$$
x=y \eta+z=y \eta+z \eta^{n}=c \eta
$$

belongs to $C \eta$. Thus we have shown that

$$
C \eta \leqq C \eta+N=C \leqq C \eta \quad \text { or } C=C \eta ;
$$

and this completes the proof of the fact that $C$ is a complement.

For a convenient enunciation of the next proposition we say that an endomorphism is normally splitting if one of its complements is a normal subgroup; and that the endomorphism is uniquely splitting if it possesses one and only one complement.

Proposition 2. Suppose that the $M$-endomorphism $\eta$ of the $M$-group $G$ is almost periodic on the normal $M$-subgroup $N$ of $G$. Then

(a) $\eta$ is a normally splitting endomorphism of $G$ if, and only if, $\eta$ induces a normally splitting endomorphism in $G / N$.

(b) $\eta$ is a uniquely splitting endomorphism of $G$ if, and only if, $\eta$ induces a uniquely splitting endomorphism in $G / N$.

(c) $\eta$ is a uniformly splitting endomorphism of $G$ if, and only if, $\eta$ induces a uniformly splitting endomorphism in $G / N$.

The statements (a) and (b) are immediate consequences of Proposition 1, and a proof of (c) may be constructed without too much difficulty by using Proposition 1 and Baer [2, Corollary to Theorem A, p. 512]. We note that this theorem constitutes a generalization of Baer [2, Theorem B].

3. The partially ordered set of extension types. It will be convenient to express the fact that $N$ is a normal and $M$-admissible subgroup of the $M$-group $G$ by saying: the $M$-group $G$ is an extension of the $M$-group $N$. This makes it possible to begin with some $M$-group $N$ and to consider all the extensions of $N$. For comparison of extensions we need the following concept.

Definition 1. The $M$-homomorphism $\eta$ of the extension $G$ of $N$ into the extension $H$ of $N$ is a normality preserving $N$ - $M$-homomorphism, if

(a) $x=x \eta$ for every $x$ in $N$; and 

$H$.

(b) $\eta$ maps normal $M$-subgroups of $G$ upon normal $M$-subgroups of

Using this concept we are able to construct the partially ordered set of extension types of the $M$-group $N$. If the $M$-groups $G$ and $H$ are extensions of the $M$-group $N$, and if there exists a normality preserving $N$-M-homomorphism of $G$ into $H$, then we say that the extension type of the extension $G$ of $N$ is less than or equal to the extension type of the extension $H$ of $N$; in symbols: $\langle N\langle G\rangle \leqq\langle N<H\rangle$. It is clear that $\langle N<A\rangle \leqq\langle N<B\rangle$ and $\langle N<B\rangle \leqq\langle N<C\rangle$ imply $\langle N<A\rangle \leqq\langle N<C\rangle$. If we supplement this definition by the further definition: $\langle N<G\rangle=\langle N<H\rangle$ if, and only if, $\langle N<G\rangle \leqq\langle N<H\rangle$ and $\langle N<H\rangle \leqq\langle N<G\rangle$ then we see easily that the extension types of $N$ form a partially ordered set. (See Baer [3] for a discussion of these concepts in a concrete situation.)

In order to be able to apply the results of the preceding sections to the problem of characterizing the extension types we are going to restrict our attention to those extensions which meet the following requirement:

(S) Every normality preserving $N$-M-endomorphism of the extension $G$ of the $M$-group $N$ is a normally splitting endomorphism (possesses a complement which is a normal subgroup).

REMARK 1. It is an immediate consequence of $\$ 2$, Proposition 2 that the extension $G$ of the $M$-group $N$ meets requirement (S), if

$\left(\mathrm{S}^{\prime}\right)$ every normality preserving $M$-endomorphism of $G / N$ is a normally splitting endomorphism of $G / N$.

We note furthermore that, as a consequence of $\$ 1$, Theorem 2 , Condition $\left(\mathrm{S}^{\prime}\right)$ will be satisfied whenever $G / N$ meets the following requirement:

$\left(\mathrm{S}^{\prime \prime}\right)$ Every element in $G / N$ belongs to at least one $L_{i}(G / N)$; and every $L_{i+1}(G / N) / L_{i}(G / N)$ is the sum of a finite number of minimal normal $M$-subgroups of $[G / N] / L_{i}(G / N)$.

Consequently the subsequent results will be applicable whenever we restrict our attention to extensions by groups $G / N$ meeting requirement $\left(\mathrm{S}^{\prime \prime}\right)$.

THEOREM 1. Suppose that $G$ and $H$ are extensions of the $M$-group $N$ and that Condition (S) is satisfied by the extension $G$ of $N$. Then $\langle N<G\rangle=\langle N<H\rangle$ if, and only if, there exist direct decompositions of the $M$-groups $G$ and $H$ :

$$
G=G^{\prime} \oplus G^{\prime \prime} \quad \text { and } \quad H=H^{\prime} \oplus H^{\prime \prime}
$$

such that $N \leqq G^{\prime}$ and there exists an $M$-isomorphism of $G^{\prime}$ upon $H^{\prime}$ which leaves invariant every element in $N$. 
REMARK 2. The existence of an $M$-isomorphism of $G^{\prime}$ upon $H^{\prime}$ which leaves invariant every element in $N$ implies that $N \leqq H^{\prime}$ and that, in the usual terminology borrowed from Galois theory, $G^{\prime}$ and $H^{\prime}$ are equivalent extensions of the $M$-group $N$.

Proof. If there exist decompositions (D) which meet our requirements, then we denote by $\gamma$ the normality preserving $N$ - $M$-endomorphism of $G$ which leaves invariant every element in $G^{\prime}$ and which maps $G^{\prime \prime}$ upon 0 ; and we denote by $\rho$ an $M$-isomorphism of $G^{\prime}$ upon $H^{\prime}$ which leaves invariant every element in $N$. Then $\gamma \rho$ is a normality preserving $N$-M-homomorphism of $G$ into $H$ so that $\langle N<G\rangle \leqq\langle N<H\rangle$; and $\langle N<H\rangle \leqq\langle N<G\rangle$ may be shown likewise. Hence $\langle N<G\rangle$ $=\langle N<H\rangle$. Thus our conditions are sufficient.

Assume conversely that $\langle N\langle G\rangle=\langle N<H\rangle$. Then there exists a normality preserving $N$-M-homomorphism $\alpha$ of $G$ into $H$ and a normality preserving $N$-M-homomorphism $\beta$ of $H$ into $G$. It is clear that $\alpha \beta$ is a normality preserving $N$ - $M$-endomorphism of $G$. Thus we may apply Condition (S) on $\alpha \beta$. Consequently there exists a complement $C$ of $\alpha \beta$ which is a normal $M$-subgroup of $G$. Furthermore, since $\alpha \beta$ leaves invariant every element of $N$, it follows from $\S 2$, Proposition 1 that $N \leqq C$. This shows that

(1) $G=C \oplus R(\alpha \beta), N \leqq C$ and $\alpha \beta$ induces an automorphism of $C$. If $x$ is in $C$ and $x \alpha=0$, then $x \alpha \beta=0$. But $\alpha \beta$ induces an automorphism in $C$. Hence $x=0$. Consequently

(2) $\alpha$ induces an isomorphism of $C$ upon $D=C \alpha$ which leaves invariant every element in $N$.

Since $C$ is a normal subgroup of $G$, and since $\alpha$ maps normal $M$ subgroups of $G$ upon normal $M$-subgroups of $H, D$ is a normal $M$-subgroup of $H$. If $x$ belongs to the cross cut of $D$ and $R(\beta \alpha)$, then $x \beta$ belongs to the cross cut of $D \beta=C \alpha \beta=C$ and $R(\beta \alpha) \beta \leqq R(\alpha \beta)$, as is easily seen. But the latter cross cut is 0 so that $x \beta=0$. Since $x$ is in $D=C \alpha$, there exists an element $y$ in $C$ such that $x=y \alpha$. Hence $y \alpha \beta=x \beta=0$. But $\alpha \beta$ induces an automorphism in $C$. Hence $y=0$ and this implies $x=0$. Consequently $D \cap R(\beta \alpha)=0$. If $h$ is an element in $H$, then $h \beta$ belongs to $G=C \oplus R(\alpha \beta)$. Consequently there exist elements $h^{\prime}, h^{\prime \prime}$ in $C$ and $R(\alpha \beta)$ respectively such that $h \beta=h^{\prime}+h^{\prime \prime}$. Since $\alpha \beta$ induces an automorphism in $C$, we have $C=C \alpha \beta$. Hence there exists an element $c$ in $C$ such that $h^{\prime}=c \alpha \beta$. Then $(-c \alpha+h) \beta=h^{\prime \prime}$ is an element in $R(\alpha \beta)$; and this implies clearly that $-c \alpha+h$ belongs to $R(\beta \alpha)$. Since $c \alpha$ belongs to $C \alpha=D$, it follows that $h$ belongs to $D+R(\beta \alpha)$. Hence we have shown that $H=D+R(\beta \alpha)$. Combining the results of this paragraph we obtain:

$$
H=D \oplus R(\beta \alpha) \text {. }
$$


But it follows from (2) that $C$ and $D$ are equivalent extensions of the $M$-group $N$. Thus these direct decompositions $G=C \oplus R(\alpha \beta)$ and $H=D \oplus R(\beta \alpha)$ are of the desired type (D). This completes the proof.

REMARK 3. Theorem 1 shows that extensions of the $M$-group $N$ which belong to the same extension type really have all the essential features of the extension in common. For if $G=G^{\prime} \oplus G^{\prime \prime}$ and $N \leqq G^{\prime}$, then $G^{\prime \prime}$ is clearly a trivial component of the extension whereas the problematic part of this extension is already contained in the step from $N$ to $G^{\prime}$. This leads us to the following concept.

Definition 2. The extension $G$ of the $M$-group $N$ is a minimal extension of $N$, if $G=G^{\prime} \oplus G^{\prime \prime}$ and $N \leqq G^{\prime}$ imply that $G=G^{\prime}$ and $G^{\prime \prime}=0$.

It is a rather obvious consequence of Theorem 1 that minimal extensions of the $M$-group $N$ which meet requirement (S) are equivalent extensions.

THeorem 2. Suppose that the extension $G$ of the $M$-group $N$ satisfies Condition (S); and that

$$
G=E^{\prime} \oplus J^{\prime}=E^{\prime \prime} \oplus J^{\prime \prime}
$$

are direct decompositions of the $M$-group $G$ such that $E^{\prime}$ and $E^{\prime \prime}$ are minimal extensions of the $M$-group $N \neq 0$. Then

(a) $E^{\prime}$ and $E^{\prime \prime}$ are equivalent extensions of the $M$-group $N$;

(b) $J^{\prime}$ and $J^{\prime \prime}$ are $M$-isomorphic $M$-groups;

(c) there exists an $M$-automorphism of $G$ which leaves invariant every element in $N$, maps $E^{\prime}$ upon $E^{\prime \prime}$ and $J^{\prime}$ upon $J^{\prime \prime}$; and

(d) $G=E^{\prime} \oplus J^{\prime \prime}=E^{\prime \prime} \oplus J^{\prime}$.

Proof. We note first that Condition (S) is satisfied by $E^{\prime}$ and $E^{\prime \prime}$, since every normality preserving $N$ - $M$-endomorphism of $E^{\prime}\left(E^{\prime \prime}\right)$ is induced by one and only one endomorphism of $G$ which maps $J^{\prime}\left(J^{\prime \prime}\right)$ upon 0 , and since the latter is necessarily a normality preserving $N$ - $M$-endomorphism of $G$.

Denote now by $\alpha, \beta, \gamma, \delta$ the decomposition endomorphisms satisfying $J^{\prime} \alpha=E^{\prime} \beta=J^{\prime \prime} \gamma=E^{\prime \prime} \delta=0$ and $x=x \alpha$ for $x$ in $E^{\prime}, x=x \beta$ for $x$ in $J^{\prime}, x=x \gamma$ for $x$ in $E^{\prime \prime}, x=x \delta$ for $x$ in $J^{\prime \prime}$.

Then $\alpha \gamma$ is a normality preserving $N$-M-endomorphism in $E^{\prime \prime}$. Since (S) holds in $E^{\prime \prime}$, there exists a direct decomposition $E^{\prime \prime}=C \oplus D$ such that $D$ is the radical of the endomorphism $\alpha \gamma$ of $E^{\prime \prime}$ and $C$ a complement. It follows from $\$ 2$, Proposition 1 that $N \leqq C$. But $E^{\prime \prime}$ is a minimal extension of $N$. Hence $C=E^{\prime \prime}$. But $\alpha \gamma$ induces automorphisms in its complements. Thus we have shown that

$\left(1^{\prime \prime}\right) \alpha \gamma$ induces an automorphism in $E^{\prime \prime}$;

and we see likewise that 
(1') $\gamma \alpha$ induces an automorphism in $E^{\prime}$.

It is clear that $E^{\prime} \gamma \leqq E^{\prime \prime}$ and $E^{\prime \prime} \alpha \leqq E^{\prime}$. Hence we have

$$
E^{\prime}=E^{\prime} \gamma \alpha \leqq E^{\prime \prime} \alpha \leqq E^{\prime} \text { or } \quad E^{\prime}=E^{\prime \prime} \alpha ;
$$

and $E^{\prime \prime}=E^{\prime} \gamma$ is shown likewise. Now one deduces readily from (1') and $\left(1^{\prime \prime}\right)$ that

(2) $\gamma$ induces an $M$-isomorphism of $E^{\prime}$ upon $E^{\prime \prime}$ and $\alpha$ induces an $M$-isomorphism of $E^{\prime \prime}$ upon $E^{\prime}$; and both these isomorphisms leave invariant every element in $N$.

The statement (a) is contained in (2). Next we prove

$$
G=E^{\prime} \oplus J^{\prime \prime}=E^{\prime \prime} \oplus J^{\prime} .
$$

If $x$ belongs to $E^{\prime} \cap J^{\prime \prime}$, then $x \gamma=0$. But $\gamma$ induces, by (2), an $M$ isomorphism in $E^{\prime}$. Hence $x=0$. Thus we have shown that

$$
E^{\prime} \cap J^{\prime \prime}=0 \text {. }
$$

If $x$ is an element in $E^{\prime}$, then $x=x \gamma+x \delta$. But $x \delta$ belongs to $J^{\prime \prime}$; and so $x \gamma$ is in $E^{\prime} \oplus J^{\prime \prime}$. Hence it follows from (2) that

$$
E^{\prime \prime}=E^{\prime} \gamma \leqq E^{\prime} \oplus J^{\prime \prime} .
$$

Hence $G=E^{\prime \prime} \oplus J^{\prime \prime} \leqq E^{\prime} \oplus J^{\prime \prime}$ or $G=E^{\prime} \oplus J^{\prime \prime}$, and the other equation (3) is shown likewise.

From (3) we deduce (b) in the stronger form:

$\left(\mathrm{b}^{*}\right)$ there exist central $M$-isomorphisms between $J^{\prime}$ and $J^{\prime \prime}$; and (a) may be strengthened likewise.

The statement (c) is a fairly obvious consequence of (a) and (b), and (d) is just a restatement of (3).

REMARK 4. To insure the existence of direct decompositions of $G$, as considered in Theorem 2, one may make for instance either of the following hypotheses:

(A) Every ascending chain of direct summands of $G$ terminates after a finite number of steps; or

(D) every descending chain of direct summands of $G$ which all contain $N$ terminates after a finite number of steps.

Remark 5. Combining Theorems 1 and 2 one sees that the extension type of an extension $G$ of $N$ is essentially characterized by the "essential component" $E$ of $G$ which is a minimal extension of $N$, provided this extension meets suitable requirements, like $(S)$ and (A) on (D). The "inessential component" $J$ is uniquely determined too (up to central isomorphisms) and is needed to characterize the equivalence class of $G$ within its extension type.

Appendix: Q-groups. Groups which are not isomorphic to proper 
quotient groups have been termed elsewhere $Q$-groups (Baer [1]). It is an important problem to decide whether all finitely generated groups are $Q$-groups. This problem is as yet unsolved. An example purporting to be a counter-example contains a fairly obvious mistake (Baer [1, p. 273, line 12]). Thus the following criterion appears to be interesting.

THEOREM. If $G$ is a finitely generated $M$-group, and if every normality preserving $M$-endomorphism of $G$ splits $G$, then $G$ is a $Q$-group.

Proof. If $N$ is a normal $M$-subgroup of $G$ such that $G$ and $G / N$ are $M$-isomorphic, then there exists an $M$-endomorphism $\eta$ such that $N$ is the kernel of $\eta$ and such that $G=G \eta$. It is clear that $\eta$ maps normal $M$-subgroups of $G$ upon normal $M$-subgroups of $G$, since every subset of $G$ is the image under $\eta$ of some subset of $G$. Thus $\eta$ is a normality preserving $M$-endomorphism of $G$; and we infer from our hypothesis the existence of a complement $C$ of $\eta$.

Since $G$ is finitely generated, there exists a finite number of elements $g_{1}, \cdots, g_{n}$ which generate the $M$-group $G$. Since every coset of $G / R(\eta)$ contains one and only one element in the complement $C$, there exist uniquely determined elements $c_{i}$ in $C$ such that $g_{i} \equiv c_{i}$ modulo $R(\eta)$. Thus every $g_{i}-c_{i}$ belongs to $R(\eta)$. Since $g_{i}-c_{i}$ are finite in number, there exists a positive integer $m$ such that $\left(g_{i}-c_{i}\right) \eta^{m}=0$ for $i=1, \cdots, n$ or $g_{i} \eta^{m}=c_{i} \eta^{m}$ for $i=1, \cdots, n$.

If $t$ is an element in $M$, then

$$
\left(t g_{i}\right) \eta^{m}=t\left(g_{i} \eta^{m}\right)=t\left(c_{i} \eta^{m}\right)=\left(t c_{i}\right) \eta^{m} \quad \text { for } i=1, \cdots, n \text {. }
$$

Thus $g_{i}$ and $M g_{i}$ are mapped by $\eta^{m}$ into $C \eta^{m}=C$, since $C$ as a complement is $\eta$-invariant. But the $M$-group $G$ is generated by the elements $g_{i}$.

Hence it follows from $G=G \eta$ that

$G=G \eta^{m} \leqq C \leqq G$ or $G=C, N \leqq G \cap R(\eta)=C \cap R(\eta)=0$ or $N=0$.

Hence $G$ is a $Q$-group, as we claimed. It follows furthermore that $\eta$ is an automorphism of $G$; and thus one deduces readily from the preceding arguments the validity of the following proposition.

Corollary. The $M$-endomorphism $\eta$ of the finitely generated $M$ group $G$ is an automorphism if, and only if, $G=G \eta$ and $\eta$ splits $G$.

REMARK. An attempt to combine the preceding theorem with $\$ 1$, Theorem 2 leads to trivial results, since finitely generated $M$-groups $G$, satisfying the conditions (a) and (b) of $\$ 1$, Theorem 2 , satisfy the 
double chain condition for normal $M$-subgroups. But an $M$-group is easily (and trivially) seen to be a $Q$-group, if it satisfies the ascending chain condition for normal $M$-subgroups.

\section{BiBLIOGRAPHY}

\section{REINHOLd BAER}

1. Groups without proper isomorphic quotient groups, Bull. Amer. Math. Soc. vol. 50 (1944) pp. 267-278.

2. Splitting endomorphisms, Trans. Amer. Math. Soc. vol. 61 (1947) pp. 508-516.

3. Extension types of Abelian groups, Amer. J. Math. vol. 71 (1949) pp. 461-490.

J. SChreIER AND S. UlAM

1. Über die Permutationsgruppe der natïrlichen Zahlenfolge, Studia Math. vol. 4 (1933) pp. 134-141.

UNIVERSITY OF ILLINOIS AND

CORNELL UNIVERSITY 